\title{
Rapid symptomatic relief of HER2-positive gastric cancer leptomeningeal carcinomatosis with lapatinib, trastuzumab and capecitabine: a case report
}

\author{
Xiao-Dong Jiao ${ }^{1}$, Chunming Ding ${ }^{2}$, Yuan-Sheng Zang ${ }^{1 *}$ and Guanzhen $\mathrm{Yu}^{2,3^{*}}$
}

\begin{abstract}
Background: Gastric cancer patients with widespread metastasis, especially meningeal metastases, have an extremely prognosis and limited therapeutic choices.

Case presentation: We reported the case of a 39-year-old male patient with HER2-positive gastric cancer with bone and meningeal metastases. He presented with multiple bone metastases and received 3 cycles of docetaxel plus S1. However, he complained with headache and imaging examinations revealed leptomeningeal carcinomatosis. FISH revealed that tumor cells in the cerebrospinal fluid were HER-positive. Herceptin was added to the regimen, but the symptoms were not relieved, the patient suffered from dizziness and nausea. The chemotherapy regimen was switched d to lapatinib (orally at $1250 \mathrm{mg} /$ day, every day), capecitabine (orally at $1000 \mathrm{mg} / \mathrm{m} 2$, bid for 2 weeks, followed by a 1-week rest interval, as 1 cycle) and Herceptin (390 mg/3 weeks). After 3 weeks of the new treatment, all the symptoms relieved. The clinical complete response was maintained for 3 months.
\end{abstract}

Conclusions: Lapatinib/Capecitabine combination therapy is an alternative treatment strategy for leptomeningeal carcinomatosis of HER2-positive gastric cancer in which trastuzumab and/or chemotherapy essentially has no effect.

Keywords: Gastric cancer, Leptomeningeal Carcinomatosis, HER2, Lapatinib

\section{Background}

Gastric cancer is the third leading cause of cancerrelated death in the world [1]. It is estimated that more than $60 \%$ gastric cancer patients harbored lymph node metastasis and/or distant metastasis [2]. Although advances in the use of combination chemotherapy regimens, the prognosis for metastatic gastric cancer (MGC) is often poor, the median overall survival (OS) less than one year [3]. Recently, the ToGA study demonstrated that targeting human epithelial growth factor receptor 2 (HER2), combined with chemotherapy, prolonged OS to 13.8 months in advanced gastric or gastro-esophageal junction cancer [4]. Trastuzumab plus chemotherapy is currently regarded as the first-line

\footnotetext{
*Correspondence: doctorzangys@163.com; qiaoshanqian@aliyun.com 'Department of Medical Oncology, Changzheng Hospital, Shanghai, China ${ }^{2}$ School of Laboratory Medicine and Life Science, Wenzhou Medical University, Zhejiang, China

Full list of author information is available at the end of the article
}

standard of care for HER2-positive MGC. However, it has several shortcomings: the development of resistance and limited ability to cross the blood-brain barrier due to its large molecular weight [5]. Therefore, a small molecule inhibitor of HER2 and the epidermal growth factor receptor [EGFR] (lapatinib) has been noted to be a promising agent for HER2-positive patients suffering from brain metastasis [6].

Here we report a case of metastatic HER2-positive gastric cancer. Most importantly, the patient developed vermis leptomeningeal carcinomatosis, a rare complication of gastric cancer with extremely poor outcome. The therapy was switched to capecitabine with dual HER2 blockade (trastuzumab and lapatinib), and intrathecal injection of methotrexate and dexamethasone. The patient responded remarkably well to this regimen, with relieved symptoms including headache, nausea, vomiting, neck resistance, gait disturbance, etc.. 


\section{Case presentation}

The clinical course was presented in Fig. 1a. A 39-year-old Chinese man presented with swelling abdomen and a high level of CEA: $465 \mathrm{ng} / \mathrm{ml}$. Electronic gastroscopy and biopsy confirmed poorly-differentiated adenocarcinoma, mixed with ring cell carcinoma, in the distal stomach. Further workup with positron emission tomographycomputed tomography (PET/CT) scan demonstrated widely metastatic disease throughout his skeleton (Fig. 1b). On 2015-4-13, he was initially treated with docetaxel (150 mg, d1, q2w), S1 (orally $60 \mathrm{mg}$, Bid, d1-10, q2w), and
Endostatin (15 mg, d1-7, q2w) for two cycles, followed by docetaxel (240 mg, d1, q3w), S1 (orally $60 \mathrm{mg}$, Bid, d1-10, $\mathrm{q} 3 \mathrm{w})$ for one cycle (2015-5-20). During the third cycle of therapy, he complained positional headache, nausea, and vomiting. He stated that the headache was located in the back side of head and was associated with standing up from a lying or sitting position. The above symptoms would relieve once he returned to a supine position. He denied vision changes, gait difficulties from weakness or ataxia, memory problems, sensory abnormalities. Physical examination revealed stable vital signs with nuchal rigidity.

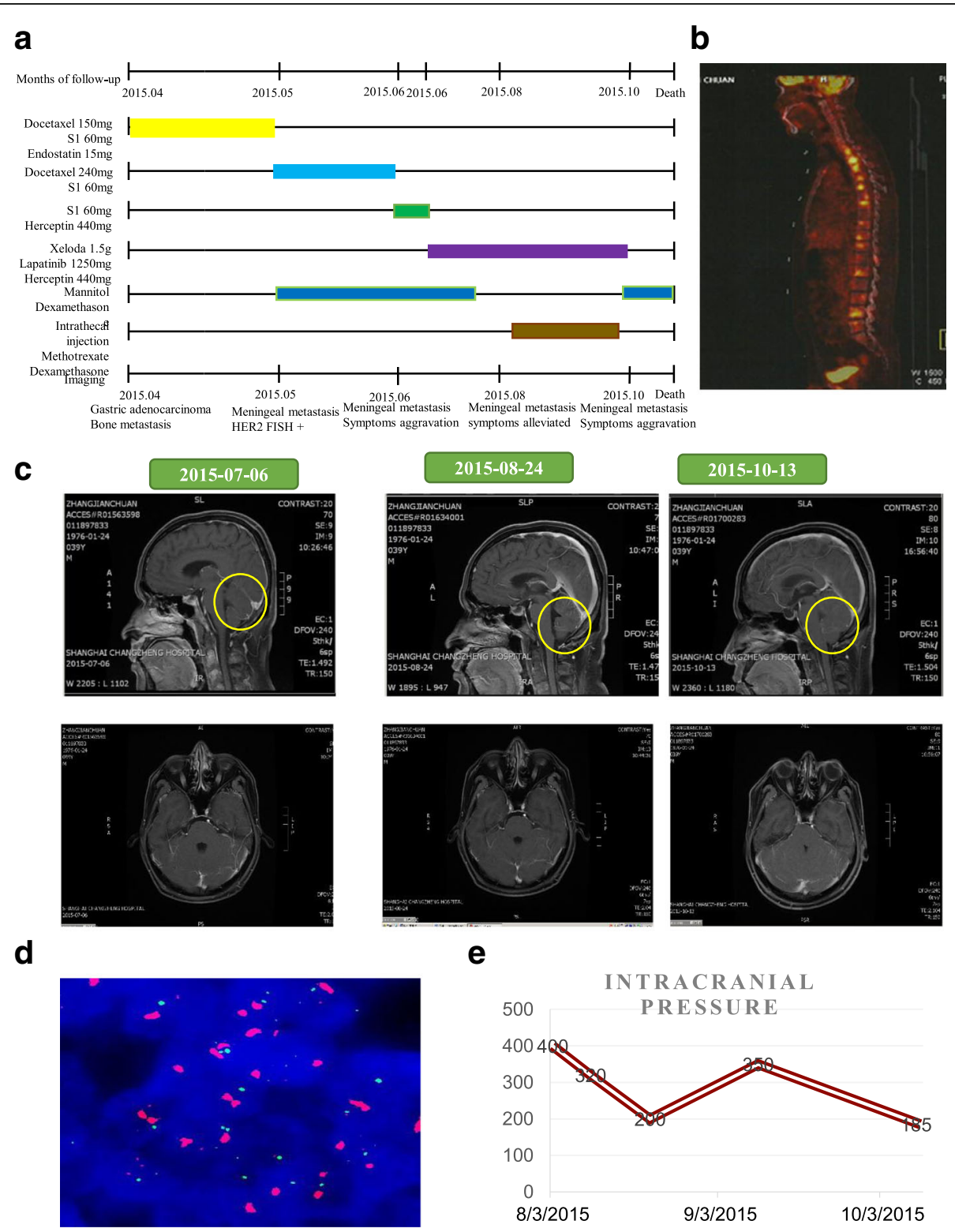

Fig. 1 The course of treatment. a Timeline describing clinical course, treatments administered and selected imaging assessments. b Positron emission tomography-computed tomography (PET/CT) scan demonstrated widely metastatic disease throughout his skeleton. $\mathbf{c}$ MRI of the brain showed edema of cerebellum at indicated time point. $\mathbf{d}$ HER2 testing of these tumor cells by fluorescent in-situ hybridization (FISH). e, The changes of intracranial pressure during the treatment process 
There was no evidence of other neurologic deficits. The patient underwent MRI of the brain and the scan revealed vermis cerebelli meningeal metastasis and edema in the vermis cerebelli (Fig. 1c). The initial lumbar puncture demonstrated a high intracranial pressure (data unknown) and found adenocarcinoma cells. HER2 testing of these tumor cells was positive by fluorescent in-situ hybridization (FISH) (Fig. 1d). Therefore, the patient was diagnosed as metastatic leptomeningeal carcinomatosis. Leptomeningeal carcinomatosis is often found in advanced gastric cancer of signet cell pathology, as in this case. On 2015-6-19, his treatment was switched to S1 and Herceptin. 2 days later, he developed vision changes and lethargy. After discussion at multi-disciplinary tumor board, on 2015-7-1, his treatment was switched to capecitabine, trastuzumab, and lapatinib. A month later, the general condition of this patient improved dramatically, and CEA in the plasma decreased notably (Table 1). Intrathecal injection of methotrexate and dexamethasone was administrated for 4 times $(2015-8-3,8-10,8-21$, and 9-10). The pressure of CSF continuously decreased when intrathecal injection was given, CEA in CSF was stable during this period (Table 2). He tolerated well and headache gradually relieved.

Along with the treatment, the whole condition of this patient turned well, intracranial pressure decreased (Fig. 1e and Table 2), nuchal rigidity dismissed, and headache relieved. Unfortunately, on October 1, he suddenly developed severe gait difficulties from ataxia, followed by unrelieved headache with vomiting, diplopia, and difficulty with speech. MRI brain revealed extremely swelling vermis cerebelli (Fig. 1c). He was taken to the emergency room and the neurosurgeon suggested surgical intervention. Given the poor outcome of this disease, the patient's family refused surgical intervention and suggested drugs to palliate his symptoms. However, the patient's performance status deteriorated progressively and died soon.

During the process of the treatment, we monitored the concentrations of $5-\mathrm{Fu}$, capecitabine, and lapatinib in the blood and cerebro-spinal fluid (CSF). Figure 2a showed that the levels of 5-Fu and capecitabine reached a peak $2 \mathrm{~h}$ after administration and declined to normal level at $10 \mathrm{~h}$, while lapatinib maintained a high level in peripheral blood. Importantly, the concentrations of capecitabine and lapatinib both maintained a relatively high level in the CSF when these drugs were administrated (Fig. 2b).

\section{Discussion and conclusions}

Leptomeningeal carcinomatosis (LC) is a rare complication of solid tumors in which the disease spreads to the membranes (meninges) of the central nervous system [7]. LC occurs in approximately 5\% of people with cancer, more common in breast, lung cancers and melanoma [8]. Very few patients with gastric cancer can develop leptomeningeal metastasis, with estimated rates of $0.16 \%$ to $0.69 \%$ [9, 10]. LC is more often observed in advanced gastric cancer of signet cell pathology, just as in this case. Due to increased intracranial pressure, most patients with LC present with severe neurologic manifestations, including headaches (usually associated with nausea, vomiting, light-headedness), gait difficulties from weakness or ataxia, memory problems, and sensory abnormalities, most of which could be observed in our patient [9]. In addition to elevated opening pressure on lumbar puncture, the protein level was elevated and the glucose level was lowed in the CSF, as in this patients [11]. MRI is a superior choice in diagnosing this disease, while CSF cytology will be necessary to confirm LC pathologically. As for our patient, MRI and CSF cytology both proved leptomeningeal carcinomatosis.

The prognosis of LC is extremely poor, if left untreated, median survival is $4-6$ weeks; if treated, median survival is 2-3 months [12]. Meanwhile, the treatment of LC is limited and intrathecal chemotherapy (methotrexate) is often suggested, but without definite curative effect, as in this patient. Interestingly, our patient presented with HER2-positive LC. The combination of trastuzumab with chemotherapy prolonged the median OS of advanced gastroesophageal cancer from under 12 to 13.8 months [4]. Because of de novo resistance and acquired resistance, only part of the patients benefited from trastuzumab therapy and the median progressionfree survival (PFS) was merely 6.7 months $[4,13]$. There are no evidences to guide clinical practice regarding trastuzumab-based chemotherapy in gastric cancer with LC. HER2-positvie breast cancer with brain metastasis appears to derive benefit from lapatinib plus capecitabine therapy [14]. Median overall survival (OS) in patients treated with lapatinib plus capecitabine was higher than in patients treated with trastuzumab-based therapy (19.1 vs. 12 months). Previous trials confirmed the safety and efficiency of the combination of trastuzumab and lapatinib in treatment-refractory patients with HER2positive metastatic cancer [15]. A synergistic effect between lapatinib and trastuzumab against HER2-positive

Table 1 CEA levels in the blood

\begin{tabular}{lllllll}
\hline & $2015-06-30$ & $2015-07-30$ & $2015-08-07$ & $2015-08-20$ & $2015-09-08$ & $2015-10-09$ \\
\hline CEA $(\mu \mathrm{g} / \mathrm{L})$ & 804.7 & 115.3 & 71.3 & 39.13 & 24 & 26.69 \\
\hline
\end{tabular}


Table 2 Components in cerebrospinal fluid of the patient with leptomeningeal carcinomatosis along with treatment of this disease

\begin{tabular}{|c|c|c|c|c|c|}
\hline DATE & 2015-08-03 & 2015-08-10 & $2015-08-21$ & 2015-09-10 & 2015-10-10 \\
\hline Intracranial pressure $\left(\mathrm{mmH}_{2} \mathrm{O}\right)$ & $>400$ & 320 & 200 & 350 & 185 \\
\hline Glucose(mmol/L) & 3.3 & 2.9 & 3.3 & 3.4 & 3.8 \\
\hline Albumen(mg/L) & 525 & 534 & 552 & 585 & 494 \\
\hline Chloride(mmol/L) & 100 & 110 & 132 & 115 & 98 \\
\hline $\mathrm{CEA}(\mu \mathrm{g} / \mathrm{L})$ & 78.12 & 78.94 & 75.03 & NA & 26.69 \\
\hline
\end{tabular}

gastroesophageal cancer has been observed in vivo and in vitro [16]. Based on these evidences, our patient was administrated with capecitabine combined with both trastuzumab and lapatinib. He tolerated and responded well to this treatment. All the symptoms induced by LC alleviated and sustained for more than three months. To the best of our knowledge, this is the first report with dual-targeted therapy with trastuzumab and lapatinib in treating gastric cancer with LC.

There are rare experiences in the treatment of meningeal carcinomatosis. The high levels of lapatinib concentration in peripheral blood and CSF gave the direct evidence that our patient responded well to lapatinib-based chemotherapy. Unfortunately, the concentration of lapatinib in CSF is only about $1 / 5$ of that in blood. This data is consistent with previous findings that average lapatinib concentration in brain metastases was only $10-20 \%$ of those in peripheral metastases [17]. Intrathecal administration of trastuzumab seems to be a safe and in some cases effective option for those HER2-positive metastatic breast cancer [18]. Our

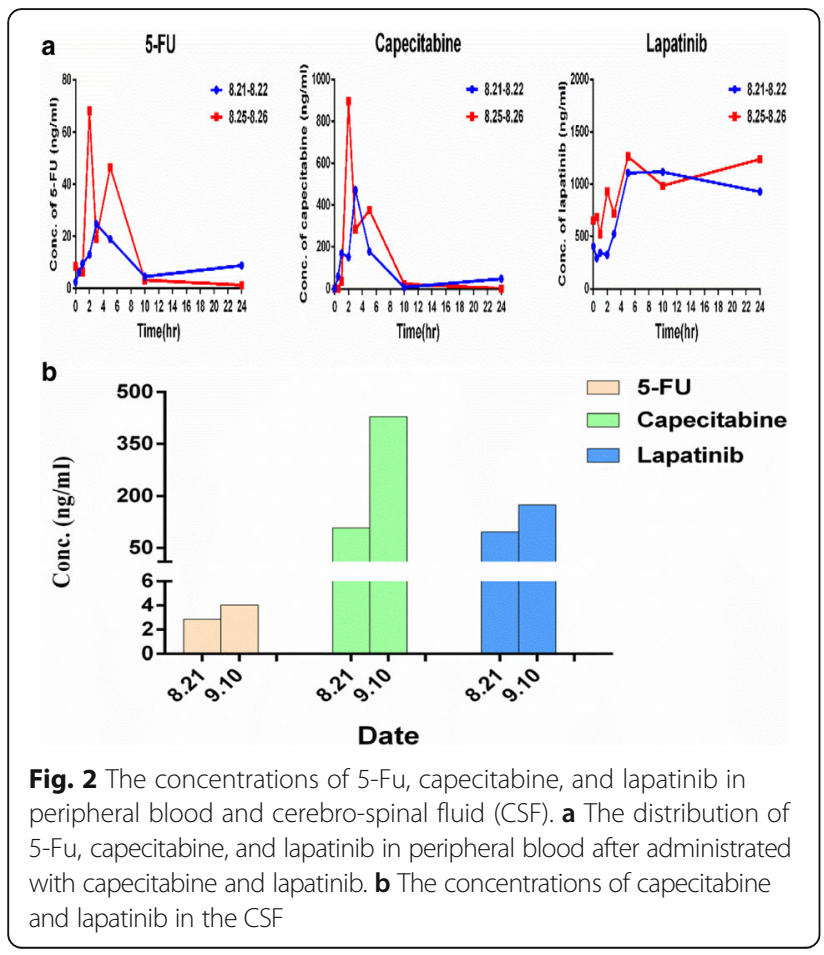

patient deteriorated rapidly when he acquired resistance to lapatinib and we didn't have to chance to carry out this option. Considering the fact that CEA level and intracranial pressure were much less than before, it is doubtable whether this patient died of acquiring resistance to HER2-targeted therapy. If so, the potential mechanisms underlying resistant to trastuzumab have been well-discussed: alteration in the HER2 receptor, loss of the tumor suppressor phosphatase and tensin homolog (PTEN), activation of various signaling pathways, immune response, etc. [19]. Whether lapatinib-resistance has the same mechanisms as trastuzumab-resistance need further study [20].

In summary, we report a case of HER2-positive gastric adenocarcinoma with rare leptomeningeal carcinomatosis and intrinsic resistance to trastuzumab-based therapy. Dual HER2 blockade appeared to be very effective in preventing LC progression. Unfortunately, the disease progressed eventually even the concentration of lapatinib sustained a relative high level. More strategies should be explored to target LC and to increase the concentration of specific drugs.

\footnotetext{
Abbreviations

CSF: Cerebro-spinal fluid; HER2: Human epithelial growth factor receptor 2; LC: Leptomeningeal carcinomatosis; MGC: Gastric cancer; OS: Overall survival; PET/CT: Positron emission tomography-computed tomography

Funding

This work was partly sponsored by Natural Science Foundation of China (81572856) and Shanghai Pujiang Program (13PJD002), International S\&T Cooperation Program of China (2014DFA33010), the Young Start-up Foundation of Changzheng Hospital (2015CZQN07), Shanghai municipal health and Family Planning Commission Foundation (201540174). The funding body had no role in the design of the study and collection, analysis, and interpretation of data and in writing the manuscript'.
}

\section{Availability of data and materials}

All data generated or analyzed during this study are included in this published article.

\section{Authors' contributions}

$\mathrm{XJ}$ and $\mathrm{YZ}$ analyzed and interpreted the patient data. CD help interpreting the data generated from the blood samples. GY wrote the manuscript. All authors read and approved the final manuscript.

Ethics approval and consent to participate

The institutional review boards of the Changzheng Hospital approved this study.

\section{Consent for publication}

The informed consent was obtained from the patient's guardian (father). 


\section{Competing interests}

The authors declare that they have no competing interests.

\section{Publisher's Note}

Springer Nature remains neutral with regard to jurisdictional claims in published maps and institutional affiliations.

\section{Author details}

'Department of Medical Oncology, Changzheng Hospital, Shanghai, China. ${ }^{2}$ School of Laboratory Medicine and Life Science, Wenzhou Medical University, Zhejiang, China. ${ }^{3}$ Department of Oncology, Longhua Hospital Affiliated to Shanghai University of Traditional Chinese Medicine, Shanghai, China.

Received: 12 December 2016 Accepted: 9 February 2018

Published online: 20 February 2018

\section{References}

1. Chen W, Zheng R, Baade PD, et al. Cancer statistics in China, 2015. CA Cancer J Clin. 2016;66:115-32

2. Yu G, Wang J, Chen Y, Wang X, Pan J, Li G, Jia Z, Li Q, Yao JC, Xie K. Overexpression of phosphorylated mammalian target of rapamycin predicts lymph node metastasis and prognosis of chinese patients with gastric cancer. Clin Cancer Res. 2009;15(5):1821-9.

3. Fang $\mathrm{N}$, Zhang $\mathrm{HQ}$, He B, Xie M, Lu S, Wan YY, Wang NR Clinicopathological characteristics and prognosis of gastric cancer with malignant ascites. Tumour Biol. 2014;35(4):3261-8.

4. Bang YJ, Van Cutsem E, Feyereislova A, Chung HC, Shen L, Sawaki A, Lordick F, Ohtsu A, Omuro Y, Satoh T, et al. Trastuzumab in combination with chemotherapy versus chemotherapy alone for treatment of HER2-positive advanced gastric or gastro-oesophageal junction cancer (ToGA): a phase 3 open-label, randomised controlled trial. Lancet. 2010;376(9742):687-97.

5. Koo T, Kim IA. Brain metastasis in human epidermal growth factor receptor 2-positive breast cancer: from biology to treatment. Radiation oncology journal. 2016;34(1):1-9.

6. Saleem A, Searle GE, Kenny LM, Huiban M, Kozlowski K, Waldman AD, Woodley L, Palmieri C, Lowdell C, Kaneko T, et al. Lapatinib access into normal brain and brain metastases in patients with her-2 overexpressing breast cancer. EJNMMI Res. 2015;5:30.

7. Mack F, Baumert BG, Schafer N, Hattingen E, Scheffler B, Herrlinger U, Glas M. Therapy of leptomeningeal metastasis in solid tumors. Cancer Treat Rev. 2016:43:83-91

8. Taillibert S, Laigle-Donadey F, Chodkiewicz C, Sanson M, Hoang-Xuan K, Delattre JY. Leptomeningeal metastases from solid malignancy: a review. J Neuro-Oncol. 2005;75(1):85-99.

9. Kim M. Intracranial involvement by metastatic advanced gastric carcinoma. J Neuro-Oncol. 1999:43(1):59-62.

10. Wasserstrom WR, Glass JP, Posner JB. Diagnosis and treatment of leptomeningeal metastases from solid tumors: experience with 90 patients. Cancer. 1982;49(4):759-72.

11. Kim NH, Kim JH, Chin HM, Jun KH. Leptomeningeal carcinomatosis from gastric cancer: single institute retrospective analysis of 9 cases. Annals of surgical treatment and research. 2014;86(1):16-21.

12. Leal T, Chang JE, Mehta M, Robins HI. Leptomeningeal metastasis: challenges in diagnosis and treatment. Current cancer therapy reviews. 2011;7(4):319-27.

13. Stern HM. Improving treatment of HER2-positive cancers: opportunities and challenges. Sci Transl Med. 2012;4(127):127rv122

14. Kaplan MA, Isikdogan A, Koca D, Kucukoner M, Gumusay O, Yildiz R, Dayan A, Demir L, Geredeli C, Kocer M, et al. Clinical outcomes in patients who received lapatinib plus capecitabine combination therapy for HER2-positive breast cancer with brain metastasis and a comparison of survival with those who received trastuzumab-based therapy: a study by the Anatolian Society of Medical Oncology. Breast cancer. 2014;21(6):677-83.

15. Sartore-Bianchi A, Trusolino L, Martino C, Bencardino K, Lonardi S, Bergamo F, Zagonel V, Leone F, Depetris I, Martinelli E, et al. Dual-targeted therapy with trastuzumab and lapatinib in treatment-refractory, KRAS codon 12/13 wild-type, HER2-positive metastatic colorectal cancer (HERACLES): a proofof-concept, multicentre, open-label, phase 2 trial. The Lancet Oncology. 2016;17(6):738-46
16. Wainberg ZA, Anghel A, Desai AJ, Ayala R, Luo T, Safran B, Fejzo MS, Hecht JR, Slamon DJ, Finn RS. Lapatinib, a dual EGFR and HER2 kinase inhibitor, selectively inhibits HER2-amplified human gastric cancer cells and is synergistic with trastuzumab in vitro and in vivo. Clin Cancer Res. 2010;16(5):1509-19.

17. Taskar KS, Rudraraju V, Mittapalli RK, Samala R, Thorsheim HR, Lockman J, Gril B, Hua E, Palmieri D, Polli JW, et al. Lapatinib distribution in HER2 overexpressing experimental brain metastases of breast cancer. Pharm Res. 2012;29(3):770-81.

18. Zagouri F, Sergentanis TN, Bartsch R, Berghoff AS, Chrysikos D, de Azambuja E, Dimopoulos MA, Preusser M. Intrathecal administration of trastuzumab for the treatment of meningeal carcinomatosis in HER2-positive metastatic breast cancer: a systematic review and pooled analysis. Breast Cancer Res Treat. 2013;139(1):13-22.

19. Luque-Cabal M, Garcia-Teijido P, Fernandez-Perez Y, Sanchez-Lorenzo L, Palacio-Vazquez I. Mechanisms behind the resistance to Trastuzumab in HER2-amplified breast cancer and strategies to overcome it. Clinical Medicine Insights Oncology. 2016;10(Suppl 1):21-30.

20. D'Amato V, Raimondo L, Formisano L, Giuliano M, De Placido S, Rosa R, Bianco R. Mechanisms of lapatinib resistance in HER2-driven breast cancer. Cancer Treat Rev. 2015;41(10):877-83.

\section{Submit your next manuscript to BioMed Central and we will help you at every step:}

- We accept pre-submission inquiries

- Our selector tool helps you to find the most relevant journal

- We provide round the clock customer support

- Convenient online submission

- Thorough peer review

- Inclusion in PubMed and all major indexing services

- Maximum visibility for your research

Submit your manuscript at www.biomedcentral.com/submit
) BioMed Central 